\title{
Structures Sédimentaires et Ichnofossiles des Formations du Crétacé Supérieur Sommital et du Tertiaire du Bassin Sud-Ouest de la Côte d'Ivoire : Intérêt Paléoenvironnemental
}

\author{
K. T. S. Amalaman, \\ K. L. Djeya, \\ J. W. D Douzo,
}

Département des Géosciences Marines, UFR des Sciences de la Terre et des Ressources Minières, Université Félix Houphouët Boigny, Abidjan, Côte d'Ivoire. Laboratoire des Géosciences et Techniques de l'Environnement,

Faculté des Sciences, Université Chouaïb Doukkali, El Jadida, Maroc

\section{W. B Dibo, N. J.P.Yao, \\ Z. B. Digbehi,}

Département des Géosciences Marines, UFR des Sciences de la Terre et des Ressources Minières, Université Félix Houphouët Boigny, Côte d'Ivoire

\section{A. Toufiq,}

Laboratoire des Géosciences et Techniques de l'Environnement, Département de Géologie, Faculté des Sciences, Université Chouaïb

Doukkali, Maroc

\section{Doi:10.19044/esj.2019.v15n33p210 URL:http://dx.doi.org/10.19044/esj.2019.v15n33p210}

\section{Résumé}

Les falaises de Fresco situées dans la partie occidentale du bassin côtier ivoirien font l'objet de la présente étude. La lithostratigraphie et la biostratigraphie des formations du Crétacé sommital et du Tertiaire, ont été revues à partir des cinq (5) coupes de terrain levées, décrites et datées. L'objectif du présent travail est de définir la dynamique d'un milieu (à une époque), appuyée sur l'identification et l'interprétation des figures sédimentaires et des ichnofossiles. Une description de synthèse proposée a permis de déduire l'intérêt paléoenvironnemental des dépôts analysés. 44 échantillons prélevés sur des coupes de cinq (5) falaises, ont servi de support à cette étude. La lithologie indique une sédimentation argileuse et sableuse dominante, avec intercalations de grès et calcaires glauconieux et phosphatés. Les structures sédimentaires décrites sont de types et d'origines variés. Ces figures apparaissent comme des formes construites par l'action d'un 
écoulement fluide, produites par les rides asymétriques et les dunes. Elles résultent de transport en masse (slumping, coulées) et de courants de turbidité. Les stratifications se présentent sous diverses formes, entrecroisées, arêtes de poissons et tabulaires, dish structures, flasers et lenticulaires. Les empreintes de déformations produites après le dépôt et avant consolidation ont été identifiées par des figures de charges, des convolutes et des structures slumpées. Sur le plan paléoenvironnemental, ces structures sédimentaires constituent de bons critères stratigraphiques et de bons indicateurs du sens du courant. Ces ichnofossiles constituant un événement post-dépositionnel, se retrouvent dans divers milieux, continentaux à marins. Ils sont généralement associés aux marges côtières et environnements marins peu profonds.

Mots-clefs : Structures sédimentaires, Ichnofossiles, Crétacé supérieur, Tertiaire, Bassin Ivoirien, Paléoenvironnements 


\title{
Upper Cretaceous and Tertiary Sedimentary and Ichnofossil Structures of the Southwest Ivory Coast Basin: Paleoenvironmental Interest
}

\author{
K. T. S. Amalaman, \\ K. L. Djeya, \\ J. W. D Douzo,
}

Département des Géosciences Marines, UFR des Sciences de la Terre et des Ressources Minières, Université Félix Houphouët Boigny, Abidjan, Côte d'Ivoire. Laboratoire des Géosciences et Techniques de l'Environnement,

Faculté des Sciences, Université Chouaïb Doukkali, El Jadida, Maroc

W. B Dibo,

N. J. P. Yao,

Z. B. Digbehi,

Département des Géosciences Marines, UFR des Sciences de la Terre et des Ressources Minières, Université Félix Houphouët Boigny, Côte d'Ivoire

\section{A. Toufiq,}

Laboratoire des Géosciences et Techniques de l'Environnement, Département de Géologie, Faculté des Sciences, Université Chouaïb

Doukkali, Maroc

\begin{abstract}
Cliffs of Fresco located southwestern part of ivorian coastal basin are subjet of this study. Lithostratigraphy of upper Cretaceous and Tertiary series were reviewed from the five (5) surveyed, described and daded. This aimed to define the dynamics of an environment (at a time), based on identification and interpretation of sedimentary figures and ichnofossils. A proposed synthesis description allowed to deduce paleoenvironmental interest of analyzed deposits. 44 samples taken from the five (5) cliffs sections upported this study. The lithology indicates a dominant clay and sand sedimentation with intercalations of sandstone and glaucanious and phosphated limestone. Sedimentary structures described are of various types. These figures appear as forms built by a fluid flow action, produced by asymmetrical wrinkles and dunes. They result from mass transport (slumping, casting) and turbidity flow; The stratifications are various shapes : intertwined, fishbone and tabular, dish structures, flasers and lenticulars beddings. Deformation imprints produced after deposition and before consolidation have been identified by load figures,
\end{abstract}


convolutes, slumped structures. The palaeoenvironmental level, these sedimentary structures are good stratigraphic criteria and good indicators of the direction of flow. These ichnofossils are post-depositional event and occur in a variety of environments, from continental to marine. They are generally associated with coastal margins and shallow marine environments.

Keywords: Sedimentary structures, Ichnofossils, Upper Cretaceous, Tertiary, Ivorian Basin, Paleoenvironments

\section{Introduction}

Sur le plan lithostratigraphique, les formations du Crétacé supérieur et du Tertiaire du bassin de Côte d'Ivoire ont fait l'objet de plusieurs travaux (Leneuf, 1968 ; Bacchiana et Laval, 1979 ; Dufaure et Tastet, 1984 ; Affian, 1986 ; Digbehi, 1987 ; Aka, 1991 ; Monde, 1997 ; Robertson, 1998 ; Yao, 2013 ;). Des études de dépôts de sondages et de coupes de terrains indiquent l'intervention de phénomènes hydrodynamiques épisodiques résultant de l'action combinée de vagues de tempêtes et de courants (tempestites). L'évolution paléoenvironnementale des falaises de Fresco commence au Maastrichtien dans un environnement marin peu profond et réducteur qui va subir peu de variations jusqu'au Mio-Pliocène (Yao, 2013). Selon les travaux de Reyre et Tea (1981) les oscillations eustatiques étaient importantes et marquées par trois périodes de transgression (Maastrichtien, Eocène inférieur et Mio-Pliocène) et trois périodes de régression (Paléocène, Oligocène et MioPliocène). Les mouvements tectoniques (failles normales) ont favorisé la mise en place des falaises vives de Fresco, par des dépôts discordants de sables calcaires sur le socle granitique birimien (Tagini, 1971 ; Yacé, 1976).

Dans ces séries, les structures sédimentaires ont été léguées au second plan alors qu'elles constituent de nos jours des diagnoses intéressantes pour la description des faciès sédimentaires pour en déduire les environnements de dépôt. C'est pourquoi la présente étude projette de décrire les formations des falaises vives de Fresco, en insistant sur les figures sédimentaires et les traces fossiles afin de mieux décrire les environnements de dépôts, car la mise en place de ces structures ou figures sédimentaires perturbe en général les couches des roches sédimentaires.

En effet, la sédimentation des fragments de roches, minéraux et fossiles se produit dans un milieu de dépôt où règnent des conditions qui le caractérisent en particulier des conditions hydrodynamiques, susceptibles d'influencer ces dépôts. De nombreuses questions nécessitent encore des réponses précises notamment, est ce que les figures sédimentaires sont observables sur les affleurements de Fresco ? Malgré la fréquence des éboulements qu'ils subissent et si oui, peut-on les identifier, les interpréter, et donc les utiliser pour reconstituer les conditions du milieu de sédimentation ? 
Par ailleurs, après le dépôt et la consolidation des sédiments, les roches ont-elles pu être soumises à des déformations de leurs strates. De ce fait, estil possible de les identifier, les caractériser ? L'objectif général est d'identifier ces structures et les utiliser pour reconstituer les conditions de formation d'un ensemble sédimentaire étudié et des conditions environnementales de dépôt par le biais des données de l'hydrodynamisme : conditions du milieu de sédimentation, conditions de déformation après consolidation.

Deux objectifs spécifiques seront visés; (i) identifier les différentes figures sédimentaires et ichnofossiles contenus dans les niveaux décrits et (ii) déterminer leur milieu de dépôt.

\section{Aperçu géologique de la zone d'étude}

Le bassin sédimentaire côtier ivoirien bordant le rivage de l'océan Atlantique depuis Sassandra à l'Ouest jusqu'à la frontière du Ghana (ville d'Axim) à l'Est, couvre une superficie de $30000 \mathrm{~km}^{2}$, et présente une partie immergée (22.000 $\mathrm{km}^{2}$ environ) et une partie émergée de $800 \mathrm{~km}^{2}$ (qui supporte la présente étude) sous forme d'un croissant centré sur Jacqueville à 50 km d'Abidjan (Spengler et Delteil, 1966). Il s'étend sur 350 km de long sur 35 à $40 \mathrm{~km}$ de large. Il ne couvre que 2,5\% du territoire ivoirien et est traversé d'Ouest en Est par une faille importante appelée « accident majeur des Lagunes ». Le rejet de cette faille oscille entre 3500 et $5000 \mathrm{~m}$. L'action de cette faille sur le bassin émergé permet de distinguer deux zones (Spengler et Delteil, 1966) :

- un compartiment nord où le socle est peu profond (170 m à Locodjro), recouvert par les dépôts du Continental Terminal (argiles, sables argileux et grès plus ou moins ferruginisés) ;

- un compartiment sud, subsident, formé d'une épaisse série sédimentaire où n'affleurent que des dépôts quaternaires (sables argileux continentaux, cordons sableux marins et dépressions plus ou moins comblées de vases organiques ou de sables blancs lessivés d'origine lagunaire).

\section{Localisation et description des sites}

Les échantillons récoltés et qui servent de support à cette étude proviennent des différentes falaises de Fresco, situées dans la partie terrestre (on-shore) entre les villes de Fresco et Sassandra (longitudes W5 $30^{\prime}$ et W $5^{\circ} 45^{\prime}$ et au Sud de la latitude N 504) à $250 \mathrm{~km}$ à $1^{\prime}$ 'ouest d'Abidjan. De 1'Est vers l'Ouest (Figure 1), cinq coupes de terrain ont été levées sur les falaises. 
Tableau 1 : Coordonnées géographiques des coupes géologiques étudiées

\begin{tabular}{|c|c|c|c|c|c|}
\hline & \multicolumn{5}{|c|}{ Coordonnées géographiques : Fuseau 30N } \\
\hline Coupe & Dassioko & Kossô & Fanti & 12 Frères & Françoikro \\
\hline \multirow{2}{*}{ Latitude N } & $5^{\circ} 2^{\prime}$ & $5^{\circ} 3^{\prime}$ & $5^{\circ} 3^{\prime}$ & $5^{\circ} 4^{\prime}$ & $5^{\circ} 4^{\prime} 35.14 "$ \\
& $25.60^{\prime \prime}$ & $32.05^{\prime \prime}$ & $41.47^{\prime \prime}$ & $19.46^{\prime \prime}$ & \\
\hline \multirow{2}{*}{ Longitude W } & $5^{\circ} 49^{\prime}$ & $5^{\circ} 42^{\prime}$ & $5^{\circ} 41^{\prime}$ & $5^{\circ} 37^{\prime}$ & \multirow{2}{*}{$5^{\circ} 36^{\prime} 19.38^{\prime \prime}$} \\
& $46.05^{\prime \prime}$ & $33.69^{\prime \prime}$ & $18.46^{\prime \prime}$ & $15.22^{\prime \prime}$ & \\
\hline Altitude (m) & 35 & 30 & 20 à 30 & 30 à 50 & 17 à 30 \\
\hline
\end{tabular}



Figure 1 : Localisation des coupes géologiques et points/d'échantillonnage

\section{Méthode d'étude}

Cette étude est basée sur le lever de chaque formation sédimentaire de cinq coupes des falaises : Françoikro, 12 Frères, Kossô, Fanti et Dassioko. Un grand nombre d'échantillons a été prélevé au changement de la lithologie, à la base et/ou au sommet des bancs, des prises de photos 
illustrent les structures et formation sédimentaires rencontrées et les ichnofossiles. Les figures sédimentaires rencontrées principalement dans les roches détritiques ont été décrites, figurées et interprétées en structures pré-, syn- et post- dépôt. Les unités stratigraphiques et lithologiques des formations sédimentaires du bassin ivoirien sont définis grâce aux travaux antérieurs notamment ceux de Yao (2013).

\section{Résultats}

\subsection{Lithostratigraphie}

Les coupes de chaque falaise de Fresco présentent en moyenne 7 à 8 unités lithologiques.

\subsubsection{Coupe de Françoikro}

- Paléocène-Eocène (Thanétien-Yprésien) :

La base de cette falaise, comprend un banc fin des argiles sableuses noires $(0,5 \mathrm{~m})$, surmontées par des argiles verdâtres (épaisseur $2 \mathrm{~m}$ et $11 \mathrm{~m}$ ) dolomitisées et/ou silicifiées avec de nombreux granules de glauconite et d'abondants débris coquillers (formations lumachelliques, foraminifères, échinodermes, dents de poissons,...).

A cassure fraîche, ces calcaires glauconieux montrent des grains grossiers à fins de la glauconite, gris foncés à noirs. Cette unité calcaire glauconieux débute par une sédimentation gréso-pélitique à traces et vacuoles de dissolution d'évaporites. Cette couche contient des bioclastes composés de spicules de spongiaires, de débris d'échinodermes, de radiolaires, de coquilles fines de lamellibranches, d'algues (Dasycladacées) et d'oolithes.

\section{- Mio-Pliocène :}

Cet intervalle se compose d'argiles bariolées silteuses $(4,5 \mathrm{~m})$. Ces argiles à faibles quantités de sables de couleur verdâtre, jaunâtre ou rosâtre, micacées et bioturbées, contient une riche faune benthique (foraminifères, lamellibranches,...) avec présence de pyrite. Des grès ferrugineux $(1 \mathrm{~m})$, surmontées par des sables argileux rouges $(3 \mathrm{~m})$. Des argiles latéritiques $(6,5 \mathrm{~m})$ se trouvant au sommet, sont non pyriteuses.

\subsubsection{Coupe des 12 Frères}

\section{- Maastrichtien :}

La base de cet intervalle se compose de grès argileux glauconieux (2,25 m), friables et très altérés, à base érosive.

- Paléocène :

Il comporte des argiles sableuses (environ 1,5 m) intercalées de sables argileux $(2 \mathrm{~m})$ qui commencent par des niveaux lenticulaires de faible épaisseur $(30 \mathrm{~cm})$. On identifie faiblement le lit d'argiles sableuses noires $(0,5$ $\mathrm{m})$. Ces dépôts de décantation sont accumulées dans des dépressions margino- 
littorales sous faible tranche d'eau. Les faciès de la partie moyenne et sommitale sont surtout des grès grano- et strato-croissants. Les dépôts sommitaux montrent des bases ravinantes et des structures conglomératiques de chenalisation de système fluviatile, ainsi qu'une intense activité biologique (pistes). L'évolution du faciès gréseux se caractérise par une association de la séquence de Lowe de grès à granoclassement normal, grossiers et mal classés (fluxotubidites), de sables moyens à pélitiques.

- Eocène :

Les niveaux superficiels de la falaise des 12 Frères sont constitués d'argiles calcaires glauconieuses à abondants débris coquilliers $(11 \mathrm{~m})$.

\section{- Mio-Pliocène :}

Des argiles bariolées silteuses $(6,5 \mathrm{~m})$ caractérisent cet intervalle, surmontées de grès ferrugineux $(1 \mathrm{~m})$ et suivies des sables argileux rouges $(4$ $\mathrm{m})$. Au sommet des argiles latéritiques $(8 \mathrm{~m})$, s'observe une passée de banc de grès. La pyrite (excepté dans le niveau latéritique) et les pellets phosphatés sont observés sur toute la coupe.

\subsubsection{Coupe de Fanti}

- Maastrichtien :

Sa base comporte des grès $(4 \mathrm{~m})$ parfois polygéniques provenant de sources différentes.

\section{- Paléocène :}

Des sables argileux $(4 \mathrm{~m})$ sont surmontés de niveaux d'argiles sableuses noires $(0,5 \mathrm{~m})$ et sables argileux $(6 \mathrm{~m})$. L'on note la présence de pyrite. Les argiles sableuses blanches et les sables argileux rouges présentent par endroits des substances noires ligneuses le long de la falaise.

\section{- Mio-Pliocène :}

Des argiles bariolées de diverses couleurs $(5 \mathrm{~m})$ suivies de grès $(1 \mathrm{~m})$ et de sables argileux rouges $(1,5 \mathrm{~m})$, surmontées par des argiles latéritiques (5 $\mathrm{m})$, avec une passée de grès et des bancs de croûtes ferrugineuses. Ces couches sont riches en pellets phosphatés et en débris carbonés.

\subsubsection{Coupe de Kossô}

- Maastrichtien :

A la base de cet étage on observe des grès ferrugineux parfois très consolidés $(5 \mathrm{~m})$, surmontés d'un banc de grès moins consolidés $(2 \mathrm{~m})$ et intensément bioturbés. On note par endroit la présence de nodules de fer.

- Paléocène :

A la base de cet intervalle s'observe une alternance de bancs de sables argileux conglomératiques $(3 \mathrm{~m})$, intercalés des argiles sableuses noires $(0,5$ $\mathrm{m})$. 
- Mio-Pliocène :

Des argiles bariolées $(5 \mathrm{~m})$, surmontées de grès ferrugineux $(2 \mathrm{~m})$ et des sables argileux rouges $(3 \mathrm{~m})$ micacée. Le sommet est recouvert par des argiles latéritiques $(6 \mathrm{~m})$.

\subsubsection{Coupe de Dassioko}

- Maastrichtien:

Il se compose de grès à ciment argileux (14,5 m), surmontés par endroit de croûte ferrugineuse $(0,5 \mathrm{~m})$.

- Paléocène:

Il s'agit de sables argileuses grise à blanchâtre $(1,5 \mathrm{~m})$, surmontés des argiles sableuses noires $(0,5 \mathrm{~m})$, suivi de sables argileux orangé à jaune $(3 \mathrm{~m})$.

- Mio-Pliocène:

Il est composé d'argile bariolée $(3 \mathrm{~m})$, suivie de grès ferrugineux (1 $\mathrm{m})$. On observe au-dessus du grès, des sables argileux rouges $(3 \mathrm{~m})$. Au sommet, des argiles latéritiques montrent quelques passées de croûtes ferrugineuses $(0,5 \mathrm{~m})$.



Figure 2 : Corrélation lithostratigraphique des falaises de Fresco

\subsection{Structures sédimentaires}

Les roches sédimentaires montrent des structures diverses qui dépendent de leur histoire. Elles sont décrites ici dans chaque coupe levée. 


\subsubsection{Falaise de Françoikro}

A la base des argiles calcaires verdâtres à lumachelles, les figures sédimentaires observées correspondent à des stratifications en mamelons (hummocky cross stratification), des sillons érosifs amalgamés ou non et des laminations distales. Leur rapide induration au niveau du fond leur confère une rigidité d'ensemble ; Elles font alors l'objet du creusement de chenaux, de ruptures sous-jacentes par bourrages (stratifications entrecroisées en feston (Fig. 3A) qui contiennent des bioclastes macroscopiques. Il a été observé des macrofaunes benthiques, et pélagiques (Nautiles et benthiques). Les rides d'interférences régulières et perpendiculaires sont observées à la surface de la formation calcaire, suivies de rides d'oscillations avec pseudomorphoses (wave-ripples) et mud-cracks. Il a été observé des flute-casts de petites tailles parfois anastomosés (Fig. 3B), des fissures de boues «mud cracks » (Fig. 3D) qui peuvent être suivies par des empreintes de pas de vertébrés, fouisseurs et d'empreintes d'origine végétale. Les figures de transport mécanique sont déterminées par la présence de figures d'objets (tool-marks) et de figures d'impact ou de rebonds, bounce casts prod casts ou prod marks (Fig. 3C). Ces objets observables sont des fragments de sédiment ou de bioclastes divers (troncs d'arbres et autres restes végétaux et animaux, coquilles, galets durs ou mous...), abandonnés par les courants sur le mur et le toit des sédiments. Les cannelures d'érosion (groove-marks) se présentent isolément ou par groupes. Leur profondeur est de l'ordre du millimètre $(0$ à $1 \mathrm{~mm})$. Ces figures coexistent parfois avec des rides de courant fortement asymétriques. Les empreintes de gouttes de pluie des alvéoles sub-circulaires de $1 \mathrm{~mm}$ de profondeur et de 0,5 à $1,5 \mathrm{~cm}$ de diamètre, ont été identifiées à la surface concave et lisse du calcaire. On note aussi la présence des racines de végétaux sur la surface d'une dalle et des pseudomorphoses abondantes. 




Figure 3 : Variétés de figures sédimentaires dans la coupe de Françoikro (stratifications entrecroisées en feston (A), flute casts anastomosés (B), bounce casts (C), fissures de boues (mud cracks) consolidés (D)).

\subsubsection{Falaise des 12 Frères}

La base de cette formation est constituée d'argilites, de siltites et de grès silteux à litage horizontal et à petites rides d'oscillation, rarement de courant, de fentes de dessiccation, de cubes de sel pseudomorphosé et de drapages d'argiles. Ces dépôts sont surtout issus de décantation; ils correspondent à des dépôts de dépressions margino-littorales sous faible tranche d'eau. On identifie la présence de groove casts, de figures de charges et slumpées. De rides de courant, parfois chevauchantes et à des litages déformés (convolute beddding), ont été identifiés. L'on observe aussi les bancs à laminations planes parallèles, soulignées parfois par la présence de micas. Dès le passage du Maastrichtien au Paléocène se manifeste des glissements syn-sédimentaires, éboulements et remaniements sous-marins.

Les faciès des argiles sableuses intercalées de sables argileux sont à évolution grano- et stratocroissante. Les dépôts sommitaux montrent des bases ravinantes et des structures de chenalisation typiques de système fluviatile, ainsi qu'une intense activité biologique (pistes). L'évolution du faciès se caractérise par la séquence de Lowe. On observe, la présence de grès grossier conglomératique (poudingues) (Fig. 4G), à litage entrecroisé en auges, en sets groupés et flute-casts anastomosés (Fig. 4H). Ces dépôts, résultant de processus de creusement et de remplissage au sein de chenaux actifs (Fig. 4E), constituent les dépôts de base de chenaux. On identifie, de grès fins à moyens, à litage tabulaire oblique, des foresets fortement inclinés, caractérisant l'accrétion latérale de barres transversales de chenaux ou les parties distales des écoulements sableux en tresse et en méandre. Dans les bancs de grès fins, 
à litage horizontal, on observe des linéations de délit et rides de courants à crêtes rectilignes, sinueuses ou lingoïdes. Les corps de ces chenaux sont à géométrie lenticulaire et à aspect emboîté. Il a été noté la présence des structures en coupelles ou structures plates (dish structures), connues dans la partie supérieure du banc argileux aplatis pris dans une matrice sableuse à couche moyenne de forme concave courbée vers le haut. Cette structure est marquée par un dépôt rapide. On observe des structures en auges de liquéfactions (Fig. 4F) et les figures d'échappement d'eau (Fig. 4I) sous la forme de tube en coupe transversale. Elles forment des protubérances (mini volcans) à la surface des bancs. Elles peuvent être engendrées par plusieurs facteurs : échappement d'eau au cours de la compaction ; tectonique synsédimentaire ou activité sismique produite dans le bassin de dépôt.



Figure 4 : Dépôts conglomératiques et figures sédimentaires dans la coupe des 12 frères (chenal désorganisé $(\mathrm{E})$, auges de liquéfaction $(\mathrm{F})$, conglomérat (poudingue) $(\mathrm{G})$, flutes casts anastomosés $(\mathrm{H})$, figures d'échappement d'eau (I)). 


\subsubsection{Falaise de Fanti}

On observe à la base du Maastrichtien, du grès micro-conglomératique montrant des rides de courants asymétriques et symétriques (Fig. 5J).

Dans la partie médiane du Paléocène, des argiles sableuses et sables argileux sont disposés en stratifications obliques montrant une discordance angulaire. Ces lits forment des crêtes des rides rectilignes sur des stratifications tabulaires (Fig. 5K) qui sont aussi des lits de plans sans mouvement, soumis à de très faibles courants. Par endroits on distingue des figures de charge (load casts) (Fig. 5L). Les formes sont variées, depuis de simples déformations locales jusqu'à des protubérances encore solidaires de la couche sableuse ou même complètement détachées. Les figures de glissements (slumps) dans les bancs argileux et sableux (Fig. 5M), fréquemment en relation avec des empreintes de charge plissotées, sont des structures de déformation du sédiment.



Figure 5 : Figures sédimentaires dans la coupe de Fanti (grès polygéniques (J), discordance angulaire : stratification oblique sur la stratification parallèle $(\mathrm{K})$, figures de charge $(\mathrm{L})$, structures slumpées et laminées (M)). 


\subsubsection{Falaise de Kossô}

La base de cette falaise se compose de grès ferrugineux parfois très consolidés, montrant des stratifications entrecroisées en festons (Fig. 6N). Ces stratifications de taille centimétrique sont formées par les vagues (mouvement oscillatoire de la houle). Les rides symétriques pures, rides d'oscillation sans migration, possèdent une structure en chevron qui montre l'aggradation verticale. On identifie la présence de nodules ferrugineux (Fig. 6O). Les grès peu consolidés, sont biorturbés. Dans les bancs de sables argileux conglomératiques et des argiles sablo-silteuses du Paléocène, l'on observe des masses de sédiments glissées sous l'action de la gravité. Les structures en convolute-lamination (Fig. 6P) sont visibles dans les faciès de turbidites au voisinage des talus sous-aquatiques. L'on note la présence des stratifications entrecroisées en forme de lentille, les stratifications en arêtes de poisson (herringbone crossbedding) (Fig. 6R). Elles sont caractérisées par la superposition de lamines obliques de sens opposé. Cette structure est produite surtout sur les plages par les inversions périodiques des courants de marée. Les dish structures (structures plates) de taille 1 à $50 \mathrm{~cm}$, sont abondantes dans cette falaise, de formes minces, de couleur foncée, concaves. La présence des silts laminés (Fig. 7Q) ascendants riches en argile. 




Figure 6 : Figures sédimentaires dans la coupe de Kossô (stratifications entrecroisées en feston $(\mathrm{N})$, nodules de fer $(\mathrm{O})$, glissement de masse (slumps) $(\mathrm{P})$, silts $(\mathrm{Q})$, stratifications en arêtes de poisson $(\mathrm{R}))$.

\subsubsection{Falaise de Dassioko}

La base du Maastrichtien est également constituée de grès à ciment argileux et présente des croûtes ferrugineuses $(0,5 \mathrm{~m})$ par endroits. On identifie des laminations entrecroisées fines, suivies de marques de rebonds (scour marks) (Fig. 7R). On observe aussi des surfaces durcies et oxydées. On note la présence de structures plates (dish structures), de nombreux litages obliques de faibles amplitudes, des rides de courant et de sillons parallèles dans le grès à dragées de quartz. L'on remarque que les dish structures varient de plat à fortement courbé. Ils ne sont pas toujours facilement discernables, faute de leurs fines laminations. Parfois, leur présence en apparence en grès massif est marquée par de petites intrusions ressemblant à des digues (2 à 5 $\mathrm{cm}$ de diamètre et $2 \mathrm{~cm}$ de longueur). Les dépôts de lits de quartz plissés sous forme tabulaire (Fig. 7S), les plis couchés concentriques suivis de slumps (Fig. 7T) se produisent dans la partie supérieure des lits de grès à ciment calcaire. 
Les stratifications convolutées (Fig. 7V) partiellement liquéfiées peu après le dépôt ont été identifiées, suivies par l'identification de nodule pyriteux dans le grès calcaire.

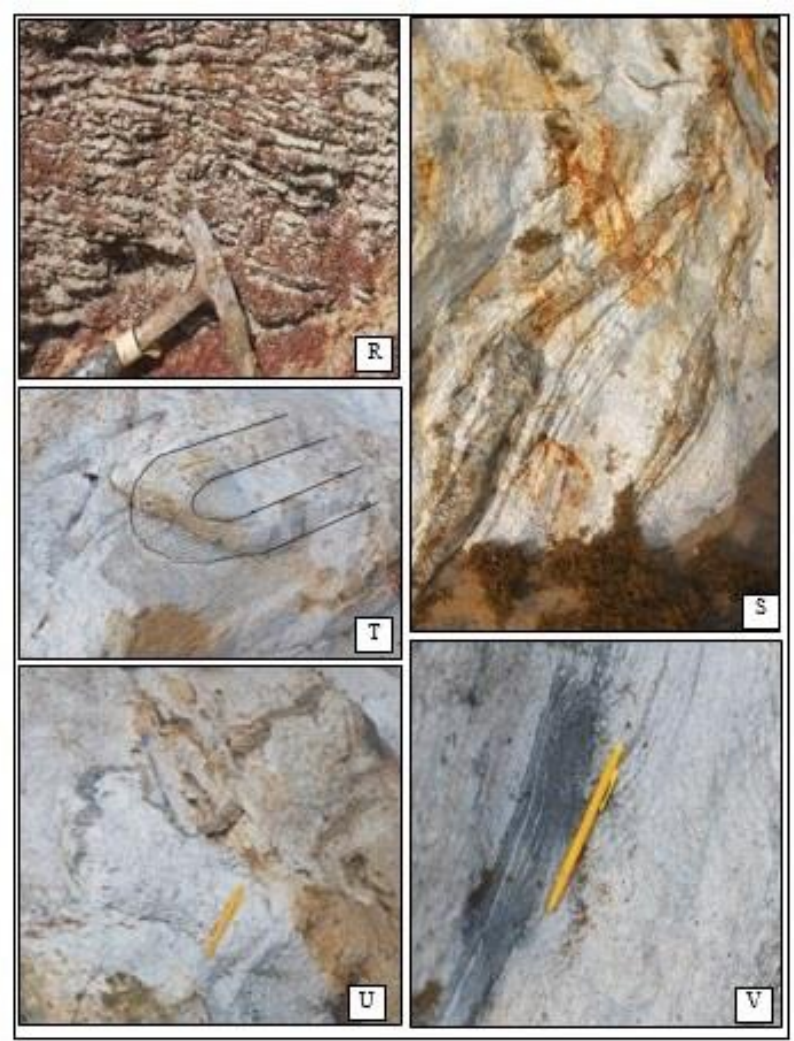

Figure 7 : Figures sédimentaires dans la coupe de Dassioko (scour marks (R), structures tabulaires (S), plis couchés concentriques (T), plis de lit de quartz (U), structures convolutes $(\mathrm{V}))$.

\subsection{Traces fossiles identifiées}

Les différentes traces fossiles observées sur les formations dans ce travail sont décrites ici et varient selon chaque coupe.

\subsubsection{Falaise de Françoikro}

Le faciès calcaire, est fortement bioturbé avec une forte intensité de traces de fossiles envahissants comprenant Thalassinoides isp. et Rhizocorallium isp. Thalassinoides isp est un système de terriers de taille relativement grande composé de parois cylindriques de 1 à $20 \mathrm{~cm}$ de diamètre. Les branches sont en forme de $\mathrm{Y}$ à $\mathrm{T}$ et avec des élargissements intermittents aux points de bifurcation. Les dimensions des terriers varient dans un système donné et les sections transversales de cylindrique, en forme de demi-lune à 
elliptique. La plupart des systèmes sont essentiellement horizontaux certains à inclinaison irrégulière (Pemberton et al., 2009; 2011). Thalassinoides isp (Fig. 8A) est généralement considéré comme un terrier d'habitation et/ou d'alimentation d'un crustacé décapode. Les points de jonction agrandis qu'ils possèdent sont souvent utilisés comme points de retournement organisme ou comme chambre de reproduction. Associé aux ichnofaciès de Cruziana dans la rive inférieure aux environnements offshore.

Rhizocorallium isp, similaire à Diplocraterion est droit, sinueux, horizontal, spreiten-terriers en forme de $U$ interprétée comme un terrier d'habitation et/ou d'alimentation d'un dépôt-alimenteur (Pemberton et al., 2009; 2011). Rhizocorallium isp se présente comme une éponge, un corail ou une algue. La présence de marques de rayures distinctes sur la paroi du tube correspond à origine des crustacés. L'on note la présence d'une forte intensité du faciès argilo-calcaire à lumachelles (Fig. 8B).


Figure 8 : Faciès fossilifères dans la coupe de Françoikro (ichnofossiles de Thalassinoides (A), argiles calcaires à lumachelles (B)).

\subsubsection{Falaise des12 frères}

La base de la formation gréseuse est érodée et présente une faible variété une bioturbations et de traces fossiles de Planolites isp, Chondrites isp, Skolithos isp et de Trypanites isp. L'ichnofaciès de Chondrites (Fig. 9C) est un système complexe de terriers ressemblant à des racines, composé de tunnels d'alimentation à ramification régulière de diamètre uniforme qui ne s'anastomosent jamais, ne s'interpénètrent pas et ne se recoupent pas. La ramification se présente généralement sous la forme de branches latérales faisant un angle supérieur à 30 degrés à 40 degrés, bifurquer aux jonctions en forme de Y (Pemberton et al., 2009; 2011).

L'ichnofaciès de Skolithos (Fig. 9D) est un élément commun de l'ichnofaciès de Cruziana. Communément associé à des vasières, il est aussi un élément commun aux dépôts d'accrétion latérale dans les chenaux estuariens (Pemberton et al., 2009; 2011). Planolites isp (Fig. 9D) est un non- 
doublé, rarement ramifié, droit à tortueux, lisse à irréguliers terriers murés ou annelés, circulaires à elliptiques en section transversale, de dimensions variables et essentiellement sans structure, différent en lithologie de la roche hôte (Pemberton et al., 2009; 2011). Trypanites isp (Fig. 9D), est un système anastomosé de perforations (éponges, bryozoaires) ou de terriers orientés perpendiculairement par rapport à la surface. Il possède une diversité faible à modérée et commun sur des surfaces indurées en zone littorale (hardground, beachrock).


Figure 9 : Faciès montrant des traces fossiles dans la coupe des 12 Frères (ichnofossiles de Chondrites (C), Sk : ichnofossiles de Skolithos, Pl : Planolites, Try : Trypanites (D))

\subsubsection{Falaise de Dassioko}

L'ichnofacies à Teredolites (Fig. 10E) se limite aux substrats ligneux. Il consiste en des forages de vers à terre souvent espacés, mais qui ne se croisent pas. Ils sont caractéristiques des terrains boisés qui représentent des surfaces d'omission développées sur des clastes de bois, des bûches de bois, des tourbes et des charbons ardents. Les producteurs typiques sont les bivalves pholadidés (tracemakers de Teredolites), bien que des crustacés (tracemakers de Thalassinoides) y soient également impliqués. Cet assemblage est associé à des surfaces d'érosion transgressives, formées en milieux marins marginaux, tels que les lagons, les baies, les estuaires et deltas (Buatois et Mángano, 2011). L'ichnofacies Zoophycos (Fig. 10F), à de très faible diversité, mais ses structures sont très abondantes. Tous ces organismes fossilisés sont des mangeurs de dépôts. Les environnements circalittoral à bathyal, marins profonds avec des conditions d'eau calme, lagons avec des argiles ou des sables argileux, sont riches en matière organique mais témoignent un milieu appauvri en oxygène. 



Figure 10 : Faciès montrant des traces fossiles dans la coupe de Dassioko (Te: Teredolites isp (E), Zoophycos isp (F)).

\section{Interprétation environnementale}

Les sédiments à grains grossiers indiquent une énergie hydrodynamique élevée. Ils sont interprétés comme des dépôts résiduels à la base de chenaux fluviatiles ((Flores et al., 1985 ; Miall, 1996)) et pourraient être considérés comme un faciès de coulée de débris. Les grès laminés rencontrés peuvent résulter de processus fluviaux (Allen 1985, Blatt et al., 1980). Courants turbiditiques (Ghibaudo, 1992) ou émaner de l'action des vagues dans l'estran vers le rivage (Cojan et Renard, 2008) et sont dans une gamme de dépôts deltaïques (front du delta, milieux intertidaux) et marins peu profonds. Le faciès conglomératique est interprété comme déposé dans le canal du front de delta proximal (Coleman 1968; Miall 1985; Wright et al., 1986; Olariu et al., 2006) et le manque d'ichnofossiles suggère un milieu à faible influence marine (MacEachern et al., 2005 ; MacEachern et Gingras 2007; Morshedian et al., 2009). La surface polygénique est formée de reliefs résultant de phénomènes d'érosion développée, dans des conditions et à des périodes différentes. Ces structures conglomératiques dynamiques témoignent d'un milieu d'assez forte énergie. Elles sont souvent associées à des figures de charges telles que les (loaded-marks) qui indiquent une action physique sur un sédiment encore gorgé d'eau.

Les lits à convolutes laminaires sont communs dans les canaux estuariens d'accrétions latérales et sont généralement interprétés comme résultant de charges dues à une sédimentation ou à un affaissement rapide. Les digues clastiques reconnues dans la zone d'étude indiquent la formation de fissures ouvertes dues à diverses causes: hydrofracturation, entailles de tension et surcharge. L'explication la plus possible de la présence des digues dans les lits de grès est la formation de fractures dans des sédiments cohésifs, qui ont 
ensuite été remplies de grès (Martín-Chivelet et al., 2011). Cette structure a été générée par une eau sous pression provoquée par une combinaison de fluidisation hydraulique (Montenat et al., 2007). La forme en feston s'observe dans une coupe perpendiculaire à la direction moyenne du courant. Les dépôts à stratification entrecroisée en feston sont dérivés de la superposition d'ensembles de courbures des lamines lors de la migration estuarienne et du chenal de marée ; ils sont également associés aux rythmes de plages et de rivages énergétiques (Cojan et Renard 2008). La présence de ces structures entrecroisées suggèrent un milieu marin peu profond caractérisé par des vagues d'orage d'activités périodiques apparentées aux dish structures (Cojan et Thiry, 1992; Cojan et Renard 2008; Li et al., 2017). Lowe et Lo Piccolo (1974) considèrent les dish structures comme des caractéristiques secondaires produites lors de la déshydratation progressive de lits rapides ou sousconsolidés.

Les structures plates évoluent, car l'eau qui s'échappe est obligée de suivre les voies d'écoulement horizontales sous des laminations semiperméables ou sous de l'eau morte piégées dans des zones de fermeture sous des laminations semi-perméables.

Les plis couchés associés et isolés observés dans la zone d'étude sont interprétés comme s'étant développés sous comportement hydroplastique à l'état quasi-solide identifié par Martín-Chivelet et al. (2011). Les structures chaotiques associées aux plis peuvent être interprétées comme preuve de liquéfaction et de fluidisation. (Montenat et al., 2007; Berra et Felletti, 2011), de stratifications convolutes irrégulières (couches déformées). Associées au complexe, les plis sont interprétés comme étant formés par fluidisation et les processus liés à l'expulsion de fluide. Ceci est probablement lié au dépôt rapide des sédiments sus-jacents. Les combinaisons de différents types de structures de déformation des sédiments meubles avec les plis allongés complexes peuvent être interprétées en termes de types de processus agissant ensemble pendant la déformation. La présence des Teredolites délimite des surfaces d'érosion transgressives coupant des dépôts de marais. Souvent indicateur important d'influence marine lorsque les Teredolites se trouvent dans des couches suggérant par ailleurs un cadre purement fluvial. Le cadre environnemental global de cette occurrence est similaire à celui de la localité classique où cet ichnofacies a été défini. Les traces de Planolites isp sont indicatrices de nutrition et de l'habitation, mais elles peuvent être trouvées dans divers environnements, (continentaux et marins) ; elles représentent des invertébrés fouisseurs, y compris les vers et les insectes (Pemberton et al., 2009; Pemberton, 2011). Ces ichnofossiles sont généralement associés à des marges côtières et milieux marins peu profonds (Hubbard et al., 2004; Pemberton et al., 2009). La présence de Chondrites isp. et de Rhizocoralium isp dans le faciès de grès indique un environnement riche en oxygène avec une 
salinité marine normale à forte (Pemberton et al., 2009; Morshedian et al., 2009), suggérant un dépôt côtier plutôt que fluvial d'eau douce. Les traces de Thalassinoides et de Trypanites suggèrent un dépôt dans un milieu marin côtier ou peu profond, avec conditions normales de salinité marine (Pemberton et Wightman, 1992; Pemberton et al., 2009; Hubbard et al., 2004; Savrda et al., 2016).

L'ichnofaciès de Skolithos est un élément commun des ichnofaciès de Cruziana (Pemberton et al., 2009; Pemberton, 2011) sur un plate-forme marine peu profonde et on le trouve souvent comme un colonisateur opportuniste de sables déposés. Aussi, est-il communément associé à des vasières et commun dans les dépôts d'accrétion latéraux de chenaux estuariens (Pemberton et al., 2009; Pemberton, 2011). La présence des Chondrites isp constituent un système de tunnels tridimensionnel à ramifications régulières comprenant une connexion ouverte à la surface et de nombreux tunnels qui se ramifient à des angles de 30 à $60^{\circ}$ pour former un motif dendritique $(\mathrm{Fu}, 1991$; Uchman, 1999). Cependant, certaines formes de chondrites isp pénètrent si profondément dans le substrat qu'elles n'auraient pu être produites que par un animaux vermiformes vivant dans la structure (Pemberton et al., 1982; 2011). La présence de Chondrites isp témoigne d'un environnement marin très salin (Morshedian et al., 2009).

Les ichnofacies de Skolithos sont caractéristiques des milieux mouvants (sable légèrement boueux à propre et bien trié) de la zone littorale, (typiques des environnements d'estran de plage et de littoral), ils sont le plus souvent développés dans des environnements marins très peu profonds.

La présence de Zoophycos isp suggère un environnement pauvre en oxygène, comme des environnements littoraux restreints à des eaux tranquilles bathyales. Ces ichnofacies de Zoophycos sont typiques des environnements offshore situés sous la base d'une onde de tempête maximale, exempts de dépôts de turbidité et de forts courants de fond $(\mathrm{Wu}, 1982$; Kotake, 1989, 2014; Richiano, 2015). Par ailleurs, ces traces sont caractéristiques d'eaux saumâtres et marines et ne sont jamais associées à l'eau douce (Pemberton et Wightman, 1992; Pemberton et al., 2009). L'intensité de la bioturbation envahissante dans ces faciès suggère un faible taux de sédimentation (Morshedian et al., 2009).

\section{Discussion}

La sédimentation dans l'ensemble des affleurements des falaises de Fresco, notamment dans la falaise des 12 Frères, nous pouvons distinguer 8 unités lithologiques (Grès argileux ; Argiles sableuses noires ; Sables argileux jaunes ; Argiles bariolées ; Grès ferrugineux ; Sables argileux rouges ; Argiles latéritiques rouges). Ce nombre diffère de ceux utilisés dans les études antérieures notamment celles de Douville (1952) qui en identifient trois, de 
Maugis (1955) alléguant une vingtaine, de Tessier (1960) et Charpy \& Nahon (1978) indiquant une quinzaine contre une douzaine pour Tastet et Roques (1969).

Ces différences sont vraisemblablement liées à des lacunes d'observations, qui seraient elles-mêmes en relation avec une altération de la qualité des affleurements (Tessier, 1960). Ces auteurs (à l'exception de Douville) distinguent plusieurs unités de lithologiques (sept à huit) dans les argiles glauconieuses à lumachelles. Le banc de grès glauconieux très altéré et friable se localise sous les falaises de Fresco, ce qui concorde avec les résultats de Douville (1952) qui ont indiqué les calcaires glauconieux à la base de l'ensemble des falaises de Françoikro.

Les structures sédimentaires identifiées dans ce travail sont nombreuses et variées telles que stratification entrecroisée, slump, stratification oblique, stratification parallèle, forme lenticulaire ou en biseau, stratification en arête de poisson, plis couchés, convolute, flute-marks, scour marks, figures de charge,...D'après les études de Vatan (1967) et Mckee et Weir (1953), ces structures sont des caractères extrêmement répandus dans les sédiments détritiques sableux ou gréseux (comme c'est le cas dans les falaises de Fresco). L'absence des argiles calcaires glauconieuses et lumachelliques dans les falaises de Dassioko, Fanti et Kossô (situées plus à l'ouest), traduit soit une sédimentation en biseau des dites couches, qui auraient été ensuite décapées par l'érosion, soit une morphologie en hauts fonds ou l'intervention de la tectonique.

Les digues clastiques similaires observées dans les couches de grès à ciment calcaire de Dassioko, ont été largement reconnues pour représenter des voies d'écoulement avec des sédiments fluidisés injectés à partir de la lithologie environnante à la suite de l'augmentation de la pression interstitielle (Daley, 1971; Berra et Felletti, 2011).

Les mécanismes de déformations tels que les plis couchés et autres sédiments associés aux structures de déformation reconnues dans cette étude peuvent être liés aux processus hydrauliques provoqués par l'afflux soudain d'eaux chargées de sédiments sur les faces glissantes des lits croisés en raison de la gravité (McKee et al., 1971).

Ces afflux pourraient également provenir du cisaillement du lit produit par la traînée d'une masse fluide riche en sédiments s'écoulant sur des sables saturés en eau (Middleton, 1966; Rosetti, 1999).

Les structures sédimentaires et les ichnofossiles dans les sédiments des falaises de Fresco indiquent un environnement de dépôt marin de faible profondeur, caractérisé par de nombreuses contraintes physiques et chimiques qui ont fortement influencé le comportement des organismes fouisseurs.

Les traces fossiles telles que les Chondrites isp désignent des salinités marines décrites dans plusieurs travaux (Pemberton et al., 1982; Hubbard, 
1999; MacEachern et al., 2005, MacEachern et al., 2007; Morshedian et al., 2009) plutôt que les conditions fluviales d'eau douce. Les Planolites isp sont caractéristiques des environnements sédimentaires dominés par les tempêtes (Dam et Andreasen, 1990) et leurs terriers se trouvent dans pratiquement tous les environnements allant de l'eau douce au milieu marin profond (Pemberton et al., 1982, 1992, 2009, 2011). Les ichnofossiles de Skolithos typiques des eaux saumâtres, souvent développés dans des environnements marins très peu profonds, ils peuvent également se former dans les conditions appropriées dans des environnements de haute mer soumis à de forts courants. (MacEachen et Pemberton 1992, 1994; Pemberton et Wigthman 1992; Pemberton et al., 2009). Les Skolithos indiquent aussi la présence de sable plus ou moins bien trié dans des environnements soumis à de fortes vagues ou à de forts courants. Ces environnements sont caractérisés par des retouches fréquentes et des changements rapides du taux de sédimentation.

Les terriers profonds tels que Thalassinoides isp sont couramment construits par des organismes tentant de tamponner les effets de la fluctuation de la salinité (qui peut aller de douce à hypersaline) résultant de l'échange de marée, plate-forme de marée, précipitation et rejet des eaux souterraines. De plus, selon Hubbard et al., (2004), la préservation de la trace d'eau saumâtre assemblages de fossiles dans des faciès boueux est compatible avec un faible taux d'oxygène dissous dans des baies interchangeables à eau calme. Les ichnofacies des Trypanites sont caractéristiques des fonds indurés tels que les côtes rocheuses, les rochers, les récifs, les lits d'ossements (Pemberton et al., 1992). Les ichnofacies de Teredolites sont caractéristiques des substrats organiques (charbons, bois fossiles, clastes de bois, etc...) et résultent de l'action de vers polychètes ou parfois de crustacés (Pemberton et al., 1992).

\section{Conclusion}

L'analyse des sédiments d'âge Maastricthien à Tertiaire (Paléocène, Eocène et Mio-Pliocène), dans des coupes (et forages) de la région de Fresco, fait apparaître une certaine évolution dans les conditions de sédimentation. Des sédiments carbonatés ou détritiques se sont déposés sur les plate-formes adjacentes aux bordures de l'océan atlantique. Des glissements, des transports en masse (olistolithes et coulées de débris) et des courants de turbidites révélés sont des marqueurs d'une importante activité tectono-sédimentaire. Les faciès de turbidites se trouvant entre les chenaux conglomératiques résultent d'un hydrodynamisme de basse énergie.

Les figures formées par érosion et sédimentation sur la face supérieure des bancs (flutes casts, prod casts, bounce marks, stratification entrecroisée, stratification oblique, rides) sont des indicateurs de la direction et du sens du courant. Les structures convolutées, les slumps, les figures de charge qui permettent le rééquilibrage des pressions dans le sédiment avec 
développement de déformations à l'interface sable/boue, ont été bien visibles dans les falaises de Kossô et Fanti, dans les couches sables/argiles. Ces structures traduisent, en somme un dépôt dans un environnement marin de faible profondeur. Le dépôt est caractérisé par de nombreuses contraintes physiques et chimiques, qui vont fortement influencer le comportement des organismes fouisseurs.

Les ichnofossiles témoignent de l'évolution du comportement de certains organismes selon les environnements. Ces ichnofossiles se retrouvent dans divers milieux, continentaux à marins, mais sont généralement associés aux marges côtières et environnements marins peu profonds.

\section{Remerciements}

Ce travail a bénéficié du soutien du Laboratoire des Géosciences et Techniques de l'Environnement (LGTE) de la Faculté des Sciences de l'Université Chouaïb Doukkali à El Jadida (Maroc). Nous remercions vivement Prof. A. El Achheb (Directeur de LGTE) et Prof. E.M Ettachfini (Responsable de l'unité Paléontologie et Stratigraphie de LGTE).

\section{References:}

1. Affian K., 1986. Incidences réciproques des structures océanique et continentale sur l'évolution du domaine ouest africain (Golfe de Guinée et Côte d'Ivoire méridionale). Thèse Doctorat 3ème Cycle, Université Bordeaux I (France), 295 p.

2. Aka K., 1991. La sédimentation quaternaire sur la marge de Côte d'Ivoire: Essai de modélisation. Thèse Doctorat d'Etat. Université Abidjan-Cocody (Côte d'Ivoire), 320p.

3. Allen J. R. L., 1985. Wrinkle marks: an intertidal sedimentary structure due to aseismic soft sediment loading. Sedimentary. Geology 41, p. 75-95.

4. Bacchiana C. \& Laval M., 1979. Etude structurale de la région nord d'Abidjan (C.I). Problèmes des déformations tardives de l'ouverture de l'Océan Atlantique. Annuaires Facultés Sciences. Université Abidjan, 15, p. 87-96.

5. Berra F. \& Felletti F., 2011. Syndepositional tectonics recorded by soft-sediment deformation and liquefaction structures (continental Lower Permian sediments, Southern Alps, Northern Italy): stratigraphic significance. Sedimentary Geology 235, p. 249-263.

6. Blatt H., Murray G \& Middleton R., 1980. Origin of Sedimentary Rocks. Prentice Hall, Englewood Cliffs, New Jersey, 782 p.

7. Buatois L. \& Mángano M. G., 2011. Ichnology. Organism-Substrate Interactions in Space and Time. Cambridge University Press, Cambridge, $347 \mathrm{p}$. 
8. Charpy N. \& Nahon D., 1978. Contribution à l'étude lithostratigraphique et chronostratigraphique du Tertiaire du bassin de Côte d'Ivoire. Université d'Abidjan, série Documentation, 35 p.

9. Cojan I., \& Renard M., 2008. Sédimentologie. Dunod, 444 p.

10. Cojan I.; Thiry M., 1992. Seismically induced deformation structures in Oligocene shallow-marine and aeolian coastal sands (Paris Basin). Tectonophysics, 206, p. 79-89.

11. Coleman J.M., 1968. Deltaic evolution. In Fairbridge Reinhold edition, Encyclopedia of Geomorphology, Reinhold, New-York, p. 255-261.

12. Daley B., 1971. Diapiric and other deformational structures in a Oligocene argillaceous limestone. Sedimentary Geology 6, p. 29-51.

13. Digbehi Z.B., 1987. Etude comparée de la sédimentation des stades d'ouverture Atlantique-Golfe de Guinée-Golfe de Gascogne. Sédimentologie, biostratigraphie. Thèse Doctorat, Université Pau (France), 366 p.

14. Douville F., 1952. Rapport géologique sur le bassin sédimentaire de Côte d'Ivoire. Rapport inédit mission de pré-reconnaissance pétrolière A.O.F.

15. Dufaure P. \& Tastet J.P., 1984. Présence de Miocène supérieur marin de plate-forme dans des sondages peu profonds d'Abidjan (C.I.). Géologie Méditerranéene, 11, p. 67-75.

16. Flores R.M., Ethridge F.G., Miall A.D., Galloway W.E., Fouch T.D., (Eds)., 1985. Recognition of fluvial depositional systems and their resource potential. SEPM special publication. Society of economic Paleontologists and Mineralogists Core Workshorp.19: 101-126 p.

17. Fürsich F. T., 1975. Trace fossils as environmental indicators in Corallian of England and Normandy. An Inter. Journal of Paleontology and Stratigraphy, Vol 8, Issue 2, p. $151-172$.

18. Ghibaudo G., 1992. Subaqueous sediment gravity flow deposits: practical criteria for their field description and classification. Sedimentology, 39, 423-454 p.

19. Hubbard S.M., Gingras M.K. \& Pemberton S.G., 2004. Paleoenvironmental implications of traces fossils in estuarine deposits of the Cretaceous Bluesky Formation, Cadotte region, Alberta, Canada. Fossils ande Strata, No.51, p. 00- 00.USA. ISSN 0300-9491. ISBN 82-16 00 37665-6.

20. Hubbard S.M., 1999. Sedimentology and ichnology of brackish water deposits in the Bluesky Formation and Ostracode zone, Peace River Oil Sands, Alberta. Unpublished Master's Thesis, University of Alberta, Edmonton. Bulletin of Canadian Petroleum Geology, p. 47

21. Kotake N., 1989. Paleoecology of the Zoophycos producers. Lethaia 22, 327-341 p. 
22. Kotake N., 2014. Changes in lifestyle and habitat of Zoophycosproducing animals related to evolution of phytoplankton during the Late Mesozoic: geological evidence for the 'benthic-pelagic coupling model'. Lethaia 47, $165-175 \mathrm{p}$.

23. Leneuf B., 1968. Le Continental Terminal, contribution à l'établissement de la notice explicative de la carte au 1/1000000 de la Côte d'Ivoire. Université Abidjan-SODEMI, n²04, 37 p.

24. Li F., Zhang H., Jing X., \& Cheng X., 2017. Paleoenvironmental analysis of the ichnogenus Zoophycos in the Lower Devonian tempestite sediments of the Longmenshan area, Sichuan, China, Palaeogeography, Palaeoclimatology, Palaeoecology, 465, (156) p.

25. Lowe D.R., Lo Piccolo L. D., 1974. The characteristics and origins of dish and pillar structures. Journal of Sedimentary Petrology 44, 484$501 \mathrm{p}$.

26. MacEachern J.A., Pemberton S.G, 1992. Ichnological aspects of cretaceous shoreface successions and shoreface variability in the western interior seaway of North America. Society of economic Paleontologists and Mineralogists Core Workshorp 17, p. 57-84.

27. MacEachern J.A., Pemberton S.G., 1994. Ichnologic aspects of incised-valley fill systems from the Viking Formation of the Western Canada Sedimentary Basin, Alberta, Canada. Society of economic Paleontologists and Mineralogists Special Publication 51, 129-157 p.

28. MacEachern J.A., Bann K.L., Bhattacharya J.P. \& Howell C.D., 2005. Ichnology of deltas; organism responses to the dynamic interplay of rivers, waves, storms, and tides; in Goisen L. . \& Bhattacharya J.P. (eds), River Deltas, Concepts, Models, and Examples, SEPM Special Publication. 83, p. 49-85.

29. MacEachern, J.A. \& Gingras M.K., 2007. Recognition of brackishwater trace fossil suites in the Cretaceous Western Interior Seaway of Alberta, Canada; in Bromely R.G., Buatois L.A., Mangano G., Genise J.F., and Melchor R.N. (eds), Sediment -Organism Interactions: A Multifaceted Ichnology, SEPM, Special. Publication. 88, p. 149-193.

30. Mckee E.D. \& Weir G.W., 1953. Terminology for stratification and cross-stratification in sedimentary rocks. Bulletin. Geological. Society. America. 69, 1033-1064 p.

31. McKee E.D., Douglas J.R. \& Rittenhouse S., 1971. Deformation of lee-side laminae in eolian dunes. Geological Society of America Bulletin 82, 359-378 p.

32. Martín-Chivelet J., Palma R.M., López-Gómez D.A. \& Kietzmann J., 2011. Earthquake-induced soft-sediment deformation structures in Upper Jurassic open-marine microbialites (Neuquén Basin, Argentina). Sedimentary Geology 235, 210-221 p. 
33. Maugis P.A., 1955. Comptes rendus des études de pré-reconnaissance pétrolière en Afrique Occidentale Française. Bulletin. de la Direction Fédérale des Mines et de la Géologie. n¹9, p. 7-95.

34. Miall A.D., 1985. Architectural elements analysis: a new method of facies analysis applied to fluvial deposits. Earth Science Reviews, 22, p. 261-308.

35. Miall A.D., 1996. The geology of fluvial deposits. Sedimentary facies, Basin analysis, and petroleum Geology. Springer, $582 \mathrm{p}$.

36. Middleton G.V., 1966. Experiments on density and turbidity currents. I. Motion of the head. Canadian Journal of Earth Sciences 3, 523-546 p.

37. Monde S., 1997. Nouvelles approches de la cartographie du plateau continental de la Côte d'Ivoire: aspects morphologiques et sédimentologiques, Thèse Doctorat, Université Abidjan Cocody (Côte d'Ivoire), $158 \mathrm{p}$.

38. Montenat C., Barrier P., Estevou P.O., Hibsch C., 2007. Seismites: an attempt at critical analysis and classification. Sedimentary Geology 196, p. 5-30.

39. Morshedian A., MacEachern J. A. \& Dashtgard S. E., 2009. Facies characterization of the Lower Cretaceous Sparky, Waseca, and McLaren Formations (Mannville Group) of West central Saskatchewan. Volume 1, Saskatchewan Geological Survey, Sask. Ministry of Energy and Resources, Paper A-9, 14 p.

40. Olariu C. \& Bhattacharya J.P, 2006. Terminal Distributary Channels and Delta Front. Architecture of River-Dominated Delta Systems. Journal of Sedimentary Research, 76, 212-233 p.

41. Pemberton S.G. \& Wightman D.M., 1992. Ichnologic characteritics of brackish water deposits. Society of Economic Paleontologists and Mineralogists Core Workshop 17, p. 141-5 167.

42. Pemberton S. G., MacEachern J. A., Gingras M. K. \& Bann K.L., 2009. Trace Fossil Atlas: The recognition of common trace fossils in cores. Unpublished Atlas, 139 p.

43. Pemberton S. G., 2011. Applied ichnology and sedimentology short course. Unpublished, $9123 \mathrm{p}$.

44. Pemberton S.G., 1982. Trace fossils from the Athabasca Oil Sands, Alberta, Canada. Science 217, 825-827 p.

45. Reyre Y. \& Tea Y. J., 1981. Quelques dinoflagellés de la falaise de Fresco (Côte d'Ivoire) : problèmes stratigraphiques, paléontologiques et bathymétriques. 8e colloques. Afrique. Micropaléontologie. ; Paris 1980. Cahiers de micropaléontologie, volume 2, 373-383 p.

46. Richiano S., 2015. Environmental factors affecting the development of the Zoophycos ichnofacies in the Lower Cretaceous Río Mayer 
Formation (Austral Basin, Patagonia). Palaeogeography Palaeoclimatology. Palaeoecology 439, p. 17 -26.

47. Robertson D., 1998. Biostratigraphie du sondage Léopard-1 dans l'Ouest de la marge d'Abidjan. Rapport non publié, 28 p.

48. Rosetti D.F., 1999. Soft-sediment deformation structures in late Albian to Cenomanian deposits, Sao Luis Basin, northern Brazil: evidence for palaeoseismicity. Sedimentology 46, 1065-1081 p.

49. Spengler A. et Delteil J. R., 1996. Le bassin sédimentaire tertiaire de Côte d'Ivoire. In: les basins sédimentaires du littoral africain du littoral africain. Ass. serv. Géology Afrique Paris, Paris 99-113

50. Tagini B., 1971. Esquisse structurale de la Côte d'Ivoire. Essai de géotectonique structurale. Thèse Doctorat, Université Lausane (France), $302 \mathrm{p}$.

51. Tastet J.P. \& Roques R., 1969. Sur quelques minéraux accessoires des falaises paléocènes de Fresco. Annuaires Université. Abidjan, Sciences, 5, 157-174 p.

52. Tessier F., 1960. Etudes paléontologiques et géologiques sur les falaises de Fresco (Côte d'Ivoire). Introduction. Annuaires Facultés Sciences Université. Dakar, 5, 35-53 p.

53. Vatan A., 1967. Manuel de sédimentologie. Edition Technique, Paris, Cedex 15, n³59,397p.

54. Wright L. D, Yang Z.-S., Bornhold B. D., Keller G. H., Prior D. B., \& Wiseman W. ,. 1986. Hyperpycnal plumes and plume fronts over the Huanghe (YellowRiver) delta front:Geo-Marine Letters, v. 6, p. 97105.

55. Wu X.T., 1982. Storm-generated depositional types and associated trace fossils in Lower Carboniferous shallow-marine carbonates of Three Cliffs Bay and Ogmore-by-Sea. Palaeogeography Palaeoclimatology Palaeoecology. 39, $189-195$ p.

56. Yacé I., 1976. Le volcanisme éburnéen dans les parties centrale et précambrienne de Fettékro en Côte d'Ivoire. Thèse Doctorat. Université., Abidjan, 373 p.

57. Yao N.J.P., 2013. Caractérisation sédimentologique, minéralogique, géochimique et biostratigraphique des falaises vives de fresco : region de grand-lahou (cote d'ivoire). Océanologie. Thèse Unique de Docteur, Université Felix Houphouet Boigny (Côte d'Ivoire), 240 p. 